\title{
From radiotracer to radiopharmaceutical: a philosophical approach
}

\author{
Stefano Boschi ${ }^{1} \cdot$ Sergio Todde ${ }^{2}$
}

Published online: 16 October 2021

(c) The Author(s), under exclusive licence to Springer-Verlag GmbH Germany, part of Springer Nature 2021

Radiotracer, radiopharmaceutical: one name, one word.

It looks unimportant in a world which depends upon communication, constantly overwhelmed by a flood of words. And then, it very often disregards the true meaning of the words and, even more, the etymology of the words which reveal their story and define their deepest meaning. And this is curiously also true for scientific language.

"Nomen omen" is a Latin adage which, literally translated, means "destiny is in the name."

In our field, the name was not just a destiny but what was believed or wanted to be attributed to these new "molecules" which (especially with the advent of PET) had a strong impact on metabolic-functional diagnostics and, more generally, on medical research.

But our main concern is the "change" from radiotracer to radiopharmaceutical, the profound reasons which induced it, and the so radical "change" of mindset experienced by our field during the first decade of the new millennium that was simply able to transform the "being" of our "molecules": an "ontological" change. Or perhaps the unveiling of a true ontology.

We used a word of philosophical relevance, ontology, because it is precisely through a philosophical approach that we will try to bring to light the reasons for this change, to "understand and interpret" it, and to suggest a possible way out.

Yes, because as Ludwig Wittgenstein (1889-1951) claimed "the aim of philosophy is to show the fly the way out of the fly bottle" [1].

This article is part of the Topical Collection on Radiopharmacy.

Stefano Boschi

stefano.boschi@unibo.it

Sergio Todde

sergio.todde@unimib.it

1 Department of Science for the Quality of Life $(\mathrm{QuVi})$, University of Bologna, Rimini, Italy

2 University of Milano-Bicocca, Tecnomed Foundation, Via Pergolesi, 33, Monza, Italy
The term "radiotracer" was preferably used in the early years, characterized by the prevalence of the radiochemistry vs the "pharmaceutical" aspects. The first decade (or maybe more) of the PET age was marked by an explosion of new radiochemistry pathways and technologies, ignited by the exciting perspective to potentially radiolabel any molecule within the pressure of time constraints.

The development of many new radiotracers occurred, new "home-made" remotely controlled systems/modules for radiochemical synthesis were developed, and new targets for producing radionuclides (mainly carbon-11 and fluorine-18) were designed and then enhanced by the visionary, brilliant idea of Hamacher [2] of replacing $\left[{ }^{18} \mathrm{~F}\right] \mathrm{F}_{2}$ with $\left[{ }^{18} \mathrm{~F}\right] \mathrm{F}^{-}$in the synthesis of $\left[{ }^{18} \mathrm{~F}\right] \mathrm{F}-\mathrm{FDG}$, thus simplifying targetry and dramatically increasing radionuclide production, synthesis yield, and radiochemical purity.

At that time, our preparations were often called "radiotracers" because their role was to "trace" metabolic pathways or to trace receptors by specific binding; moreover, the concentration of the molecules in terms of administered mass was unbelievably low, thus further justifying the term "tracer." The concept of medicinal product was still a long way off.

Thinking back to those roaring years, we are led to refer to a philosophical category that takes us back to Friedrich Nietzsche (1844-1900) in "The Birth of Tragedy" [3]: the Dionysian.

Even though we are dealing with science, inherently linked to the concept of scientific methodology and therefore intrinsically of "truth," the atmosphere of that period was experienced by many scientists as a joyful, "free," unrestrained, instinctual developmental, and, mostly, unregulated.

A few images of the first production of $\left[{ }^{18} \mathrm{~F}\right] \mathrm{F}-\mathrm{FDG}$ in Brookhaven in 1976 of the pioneering technological approaches (Fig. 1) showed clearly how much everything was entrusted to a strong impulse and to a renewal drive that did not yet perceive the regulatory and normative burden that a few years later began to manifest and consolidate in daily 

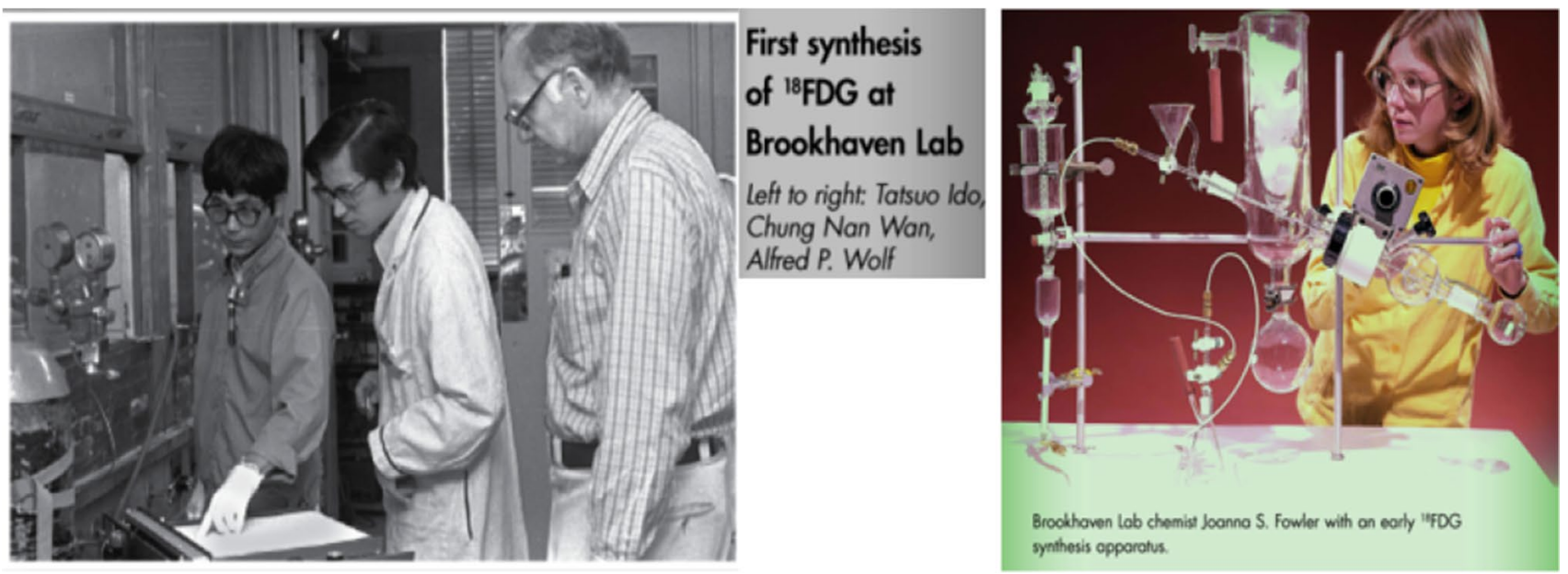

Fig. 1 Production of $\left[{ }^{18} \mathrm{~F}\right] \mathrm{F}-\mathrm{FDG}$ in Brookhaven

practice: it was "radiochemistry" which was predominant at that time, and, therefore, the product was a "radiotracer."

As in the "Birth of Tragedy," some "Socrates" and "Euripides" introduced in the field of radiochemistry a normative, regulatory, rationalist, theoretical force that we could define, by contrast, as Apollonian. The clash of these two forces, Dionysian and Apollonian, blasted in the first decade of the new millennium. Opposing energies and proud dialectical oppositions were not always aimed at integration but often at the predominance of one over the other.

"Radiochemistry" was "jeopardized" by "radiopharmacy," strengthened by its regulatory power in the background, which already viewed in the radiotracer the essence of a radiopharmaceutical, to be subjected to all the regulatory and quality restrictions imposed by its pharmaceutical "nature," without necessarily distinguishing the peculiarities and the differences between a radiopharmaceutical and a conventional drug.

Radiochemists have always vividly denounced, like Nietzsche in the "Birth of Tragedy," that this regulatory and normative excess, this Apollonian power, would lead to an impoverishment of the potential of science, of its creative capacity, and of its relationship with the knowledge of the true mechanisms that underlie the "creation" of a molecule. Only a renaissance of the primordial "Dionysian spirit" would have allowed continuing on the wave of the enormous progress reached in the early phase.

In the end, that probably happened, and the need to comply with quality and regulatory issues has undoubtedly led to a slowdown in the growth, design, and especially in the trials of new radiopharmaceuticals.

However, it is interesting to have a more "theoretical" and philosophical approach, in order to analyze what has happened, foresee what could happen, and, above all, plan and be prepared to manage a future that is already present.
We must try to dispel, and the concept is certainly not limited to our "small" area of interest, what Martin Heidegger (1889-1976) claimed about the problem of the dominant technique in modern society: "What is truly unsettling is not that the world is transformed into a complete domain of technology. Far more disturbing is that man is not at all prepared for this radical change in the world" [4].

Are we absolutely certain about our "full" understanding of the "essence" of the "structure" we have called radiotracer, radioligand, or radiopharmaceutical? Very few efforts have been made to understand this theoretical aspect, beyond sharing both corporate positions and excessive inference from pharmaceutical authorities.

Edmund Husserl (1859-1938), who established the school of phenomenology argued that we must return "back to the things themselves."

So let's go back to our nice molecules, free from any other constraint: what are we dealing with? We are dealing with a medicinal product, as reported, for instance, by the European Union 2001/83/EC directive, Art.1: Medicinal product: any substance or combination of substances presented for treating or preventing disease in human beings. Any substance or combination of substances which may be administered to human beings with a view to making a medical diagnosis or to restoring, correcting or modifying physiological functions in human beings is likewise considered a medicinal product.

If we go "back to the things themselves," following Husserl phenomenology, we can consider a radiopharmaceutical as a "structured whole" constituted of two parts: (1) chemistry, for which the concerns are chemical structure, receptor/target interactions, kinetics, synthesis, stability, etc., and (2) the regulatory and quality assurance aspects, which assure that these molecules possess proven quality, safety, 


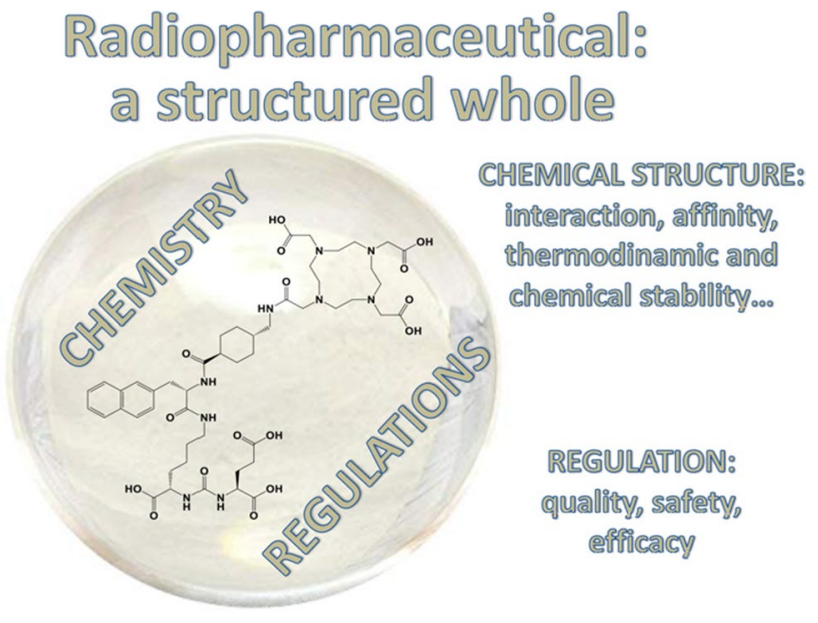

Fig. 2 Radiopharmaceutical as a "structural whole"

and efficacy characteristics to be considered medicinal products (Fig. 2).

Husserl and the Gestalt psychologists emphasized that organisms perceive entire patterns or configurations, not merely individual components and that "the whole is more than the sum of its parts."

According to Husserl, a structured whole is not a rigid structure but allows "discrete" variations of the elements. Such variations are always possible but within certain constraints beyond which the whole, as such, is destroyed.

The structure is nothing more than a set of restrictions (the so-called "the gift of constraints") to possible variations, while the variation of the elements of an integral whole may be possible within certain limits beyond which the whole is destroyed.

The typical example is a melody: if we transpose the sounds into another register, within a set of constraints, the melody remains present and recognizable. If the change is chaotic, the melody becomes cacophony.

If we meditate on this model, how can we forget the epochal changes of the last 10-15 years, with the detrimental consequences due to the variation of the elements of the integral whole due to overburdening regulations, with the risk of de-structuring the whole?

This is indeed what happened with the enforcement of increasingly stringent regulations as well as of the quality assurance systems conceived for the pharmaceutical industry (GMP) and compulsorily transferred to the world of radiopharmaceuticals, which are certainly a very different reality.

The initiation of prospective trials in nuclear medicine is especially complex because applicable guidelines are not only the good manufacturing practice (GMP) guidelines for investigational radiopharmaceuticals' clinical trials (or other specific national QA systems) but also guidelines for the investigational medicinal product dossier (IMPD),
Investigator's brochure, good clinical practice (GCP), and, to some extent, good laboratory practice for preclinical testing in case of "first-in-human" radiopharmaceuticals, not to mention the need for Ethics Committee approval and the applicable legislation on radiation protection, all of which make radiopharmaceutical preparations certainly among the most regulated.

Regulatory overload led to a decrease of competitiveness of the European Union compared to other countries with more flexible regulatory framework, like the USA, where more clinical trials have been approved in comparison with the "highly regulated" European Union [5].

How can we rebalance the set of constraints in the peculiar type of structural whole named radiopharmaceutical?

Again, understanding philosophy can lead us to a way out and can help the fly to fly out of the bottle!

Hans-Georg Gadamer (1900-2002) affirmed that every understanding of the single element is conditioned by the understanding of the whole, and each explanation of the single element presupposes an understanding of the whole.

Chemistry, like physics, is an empirical science where the empirical means "based on observation or experience": this process is a crucial part of the scientific method.

Regulations, laws, and jurisprudence belong to human and social sciences which typically require understanding and interpretation. Friederich Schleiermacher (1768-1834) affirmed that "there is interpretation in every act of understanding." The theory and methodology of interpretation is called "hermeneutics."

Hermeneutics is more than only the interpretative principles or methods used when immediate comprehension fails, it also includes the art of understanding and communication.

The concept of hermeneutics includes legal hermeneutics which can be seen as a branch of philosophical hermeneutics, whose main author in the twentieth century was HansGeorg Gadamer.

The hermeneutic approach, when applied to radiopharmaceutical regulations, might enable a proper interpretation starting from understanding the peculiar "structured whole" called radiopharmaceutical and could rebalance the set of constraints.

We are in favor of a hermeneutic approach capable of combining the "essence" of the law with the specific needs of radiopharmaceuticals, avoiding the stiffness of a merely "pharmaceutical" application; in other words, transposing the sounds to a proper register, going back to Husserl's example.

The principle of the understanding and interpretive method (often called "hermeneutic circle") could lead to different methodologies of interpretation tailored to specific situations, which, in turn, should lead to a decrease in the bureaucratic burden, increase flexibilities, and expand the 
possibility to submit new clinical trials, thus actively promoting the development of molecular imaging.

An example of "hermeneutics" in the radiopharmaceutical regulation is the shared protocol for the IMPD development of PSMA-11 administration issued by the National Regulatory Agency of France in collaborations with the applicants [6]. In most of the countries, each applicant should submit a separate investigational dossier and IMPDs for the same radiopharmaceuticals (e.g., PSMA), and each dossier should be approved separately by the regulatory agency, instead of applying according to a shared protocol. A collaboration between regulatory authorities and relevant scientific societies could contribute to design less biased studies [7]. Again, the hermeneutic approach to radiopharmaceutical regulation along with the scientific "strength" of scientific communities could better define the "structured whole."

Another example of hermeneutics may be found in the different approaches of the EU National Authorities in terms of applicable quality assurance systems, requisites for responsible of preparation, conditions to allow radiopharmaceuticals administration in humans, etc.

Indeed, one of the goals of the new EU Regulation 536/2014 for clinical trials is to harmonize the application procedures from the various EU members and possibly to simplify and speed up the procedures themselves through a centralized "portal" for trial submissions and faster assessment; moreover, it states that preparation of investigational radiopharmaceuticals for diagnostic purposes do not require several requisites (GMP compliance, site authorization, QP) anymore, thus moving in the right direction. However, this regulation's effective starting date has been heavily delayed, and "practical" feedback is not yet available. Moreover, despite of its inherent character of "firm law," which implies its direct implementation "without translation," there will probably be still room for interpretation (hermeneutics is back again!), e.g., in quality assurance requisites or the extent of documentation, so a real harmonized approach will require further additional efforts.

In conclusion, the Dionysian era of radiotracers has most probably come to an end, and they are now for everyone "radiopharmaceuticals." This transformation configures the radiopharmaceutical as a "structured whole" with two very clear and defined components, chemistry and regulations, where the variation of the elements may be possible within certain limits beyond which the whole is destroyed.
Regulatory overload could represent a risk of overcoming these limits.

Only a process of understanding and interpretation, a hermeneutics of the regulatory requirements could rebalance the constraints and maintain the "essence" of the "radiopharmaceutical," encouraging therewith the development of new clinical trials and thus pushing research towards new horizons.

\section{Declarations}

Ethical approval Institutional Review Board approval was not required because the paper is an editorial.

Informed consent Not applicable.

Conflict of interest The authors declare no competing interests.

\section{References}

1. Wittgenstein L. Philosophische Untersuchungen. 1953

2. Hamacher K, Coenen HH, Stöcklin G. Efficient stereospecific synthesis of no-carrier-added 2-[18F]-fluoro-2-deoxy-D-glucose using aminopolyether supported nucleophilic substitution. J Nucl Med. 1986;27(2):235-8.

3. Friedrich Nietzsche, Die Geburt der Tragödie aus dem Geiste der Musik 1872

4. Heidegger M: Gelassenheit. 1959

5. Clinical Trials.gov. https://clinicaltrials.gov/ct2/results/map? term $=$ PET\&map $=$. Accessed 2 Oct 2021

6. Chevalme YM, Boudali F, Gauthé M, et al. Survey by the French Medicine Agency (ANSM) of the imaging protocol, detection rate, and safety of ${ }^{68} \mathrm{Ga}$-PSMA-11 PET/CTin the biochemical recurrence of prostate cancer in case of negative or equivocal ${ }^{18}$ F-fluorocholine PET/CT: 1084 examinations. Eur J Nucl Med Mol Imaging. 2021;48:2935-50. https://doi.org/10.1007/ s00259-020-05086-1.

7. Giammarile F, Fanti S. Comments to "Survey by the ANSM of the imaging protocol, detection rate, and safety of 68Ga-PSMA-11 PET/CT." Eur J Nucl Med Mol Imaging. 2021;48:2690-1. https:// doi.org/10.1007/s00259-021-05292-5.

Publisher's note Springer Nature remains neutral with regard to jurisdictional claims in published maps and institutional affiliations. 